\title{
Leaky Opto-Electrical Neural Probe for Optical Stimulation and Electrochemical Detection of Dopamine Exocytosis
}

Vasudevan, Shashank; Kajtez, Janko; Heiskanen, Arto; Emnéus, Jenny; Keller, Stephan Sylvest

Published in:

Proceedings of the 2020 IEEE 33rd International Conference on Micro Electro Mechanical Systems (MEMS)

Link to article, DOI:

10.1109/mems46641.2020.9056270

Publication date:

2020

Document Version

Peer reviewed version

Link back to DTU Orbit

Citation (APA):

Vasudevan, S., Kajtez, J., Heiskanen, A., Emnéus, J., \& Keller, S. S. (2020). Leaky Opto-Electrical Neural Probe for Optical Stimulation and Electrochemical Detection of Dopamine Exocytosis. In Proceedings of the 2020 IEEE 33rd International Conference on Micro Electro Mechanical Systems (MEMS) (pp. 388-391). [9056270] IEEE. International Conference on Micro Electro Mechanical Systems

https://doi.org/10.1109/mems46641.2020.9056270

\section{General rights}

Copyright and moral rights for the publications made accessible in the public portal are retained by the authors and/or other copyright owners and it is a condition of accessing publications that users recognise and abide by the legal requirements associated with these rights.

- Users may download and print one copy of any publication from the public portal for the purpose of private study or research.

- You may not further distribute the material or use it for any profit-making activity or commercial gain

- You may freely distribute the URL identifying the publication in the public portal 


\title{
LEAKY OPTO-ELECTRICAL NEURAL PROBE FOR OPTICAL STIMULATION AND ELECTROCHEMICAL DETECTION OF DOPAMINE EXOCYTOSIS
}

\author{
Shashank Vasudevan, Janko Kajtez, Arto Heiskanen, Jenny Emnéus and Stephan S. Keller* \\ Technical University of Denmark, Lyngby, DENMARK
}

\begin{abstract}
This paper reports on the fabrication and characterization of leaky opto-electrical neural probes for optical stimulation and real time electrochemical detection of dopamine exocytosis from optogenetically modified neural stem cells. Indentations were introduced in a SU-8 waveguide structure, patterned directly on the probe shank, to allow light to leak over a large area. Pyrolytic carbon electrodes fabricated on both sides of the leaky waveguide allow for real time detection of dopamine. The electrochemical characterization of the pyrolytic carbon demonstrates excellent conductivity and suitability for dopamine detection.
\end{abstract}

\section{KEYWORDS}

Optical neural probe, leaky waveguide, optogenetics, electrochemistry, dopamine detection

\section{INTRODUCTION}

The ability of stem cells to differentiate into any cell type in the body makes them an excellent candidate for neurodegenerative disease therapy. Neurons with specific phenotypes, such as those releasing dopamine have been generated from various types of stem cells [1]. Transplantation of stem cell derived neurons can replace degenerated ones and enhance the production of neurotransmitter. The possibility of regulating neurotransmitter release is particularly relevant for diseases such as Parkinson's disease (PD). For instance, it is possible to provide a nearly continuous release of dopamine from dopaminergic neurons, which has been shown to minimize motor complications in PD [2].

In the last decade, optogenetics has allowed selective modulation of neural activity by illuminating neurons with a specific wavelength of light. This is achieved by genetically modifying neurons to express specific light sensitive proteins called opsins. Channelrhodopsin-2 (ChR-2) is one such non-specific cation channel used to depolarize the neuron [3]. Optical stimulation of neurons offers high temporal and spatial resolution when compared to electrical stimulation. Moreover, it is possible to modulate neurotransmitter release from optogeneticallymodified neurons [4]. However, optogenetic modulation of neurotransmitter release directly in the brain necessitates uniform illumination of a large neuronal population to achieve a therapeutic effect.

A large variety of implantable neural probes using waveguides to deliver light to various regions of the brain has been developed. A multipoint side-emission device using multiple silicon oxy-nitride waveguides ending with corner mirrors has been reported to direct light laterally with respect to the implant direction [5]. Alternatively, a
3D array of sharpened waveguides allows for stimulation at two different depths [6]. Tapered optical fibers with multiple optical windows realized by focused ion beam milling have been developed for optical stimulation at specific sites along the taper by modifying the input light coupling angle [7]. However, multipoint emitting devices need a higher injection power to achieve effective optogenetic control of neural activity of large cell populations and the efficiency depends on the distance between the active window and the waveguide tip. It is possible to enlarge the volume of activation by increasing input light intensity. However, this intensity must be below the tissue damage threshold.

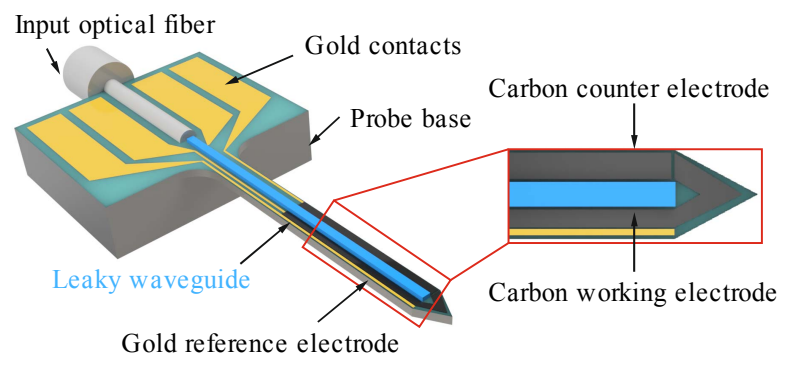

Figure 1: Schematic of the proposed opto-electrical neural probe containing a centered leaky SU-8 waveguide. Two pyrolytic carbon electrodes surround the waveguide acting as working and counter electrodes. A gold electrode serves as pseudo-reference electrode for electrochemical detection. The fourth gold contact pad is present due to the author's obsession for symmetry.

Here we report on the fabrication and characterization of an opto-electrical neural probe containing a leaky optical waveguide and pyrolytic carbon electrodes capable of optical stimulation and real-time electrochemical detection of dopamine exocytosis from a population of optogenetically modified dopaminergic neurons (Figure 1). For the definition of counter electrode (CE) and working electrode (WE) on the probe shank, the negative epoxy photoresist SU-8 was patterned using UV photolithography and subsequently pyrolysed at high temperature in an inert atmosphere. Pyrolytic carbon has earlier been shown to have excellent electrochemical properties for dopamine electrochemistry [8]. A SU-8 leaky optical waveguide was designed to distribute light over a broad volume with the aim to illuminate uniformly a large population of neural stem cells surrounding the waveguide. The probes were fabricated using microfabrication techniques. The intensity of light emitted from the leaky optical waveguide was found to be sufficient for optogenetic stimulation. Finally, the suitability of pyrolytic carbon for dopamine detection was analyzed using cyclic voltammetry. 


\section{FABRICATION}

The overall process flow for the fabrication of the opto-electrical probes is shown in Figure 2. A $1 \mu \mathrm{m}$ thick $\mathrm{SiO}_{2}$ layer was thermally grown on a $350 \mu \mathrm{m}$ thick p-doped $\mathrm{Si}$ wafer. The thick oxide cladding layer ensures decay of evanescent waves and provides electrical insulation for the electrodes.

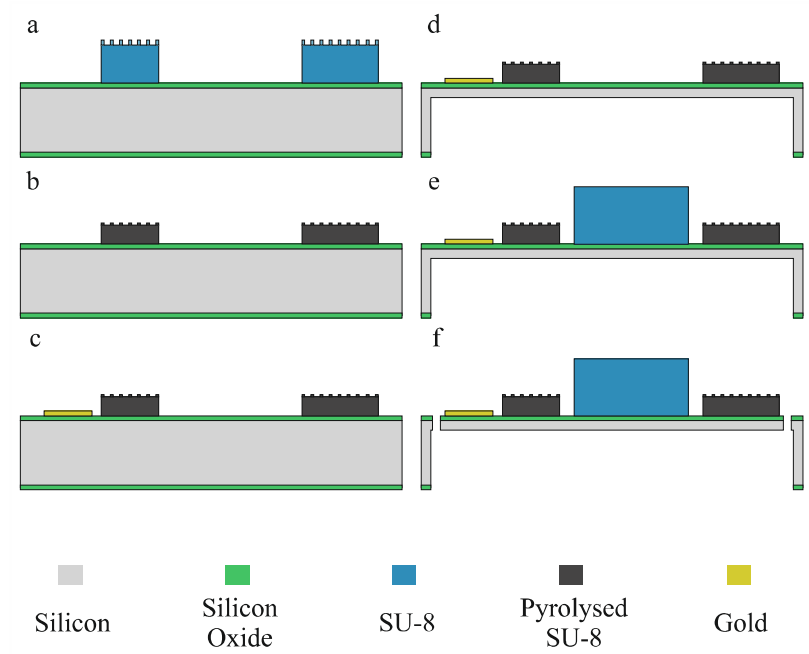

Figure 2: Cross sectional schematic of neural probe fabrication and release. a) Patterning of SU-8 by maskless UV photolithography. b) Pyrolytic carbon electrodes after pyrolysis. c) Deposition of gold pseudo-reference electrode. d) Backside DRIE to define probe thickness. e) Patterning of leaky SU-8 waveguide. f) Front side DRIE to pattern fiber groove and for probe release.

The first step involves the fabrication of pyrolytic carbon electrodes through the pyrolysis of SU-8. A $17 \mu \mathrm{m}$ thick SU-8 layer (SU-8 2035, $120 \mathrm{~s}$ at $5000 \mathrm{rpm}$ ) was patterned with UV maskless aligner $\left(250 \mathrm{mJcm}^{-2}\right.$ using Heidelberg MLA100) to define the base layer. High temperature baking of SU-8 during lithography resulted in thermal stress and subsequent cracking of the SU-8 layer. We observed that the SU-8 does not reflow during pyrolysis process to repair the cracks. The presence of cracks in the carbon severely decreases the conductivity of the layer leading to poor electrochemical response. Hence, low soft and post-exposure baking temperatures (15 min at $50{ }^{\circ} \mathrm{C}$ ) were used. On top of the first SU-8 layer, an additional $5 \mu \mathrm{m}$ thick layer of SU-8 2005 was spin coated at $5000 \mathrm{rpm}$ for $60 \mathrm{~s}$ and pillars were patterned in the same way as the base layer (Figure 2a). Following the development in PGMEA for 15 minutes and rinsing in 2propanol, the wafer was pyrolysed in $\mathrm{N} 2$ atmosphere for one hour at $900^{\circ} \mathrm{C}(\mathrm{PEO}-604$, ATV Technologie) resulting in the formation of pyrolytic carbon. The temperature during heating and cooling was ramped at $2{ }^{\circ} \mathrm{Cmin}^{-1}$. During pyrolysis, the polymer shrinks to a $2 \mu \mathrm{m}$ thick pyrolytic carbon layer (Figure 2b). A thicker layer of pyrolytic carbon would decrease the resistance of the electrodes. However, too thick a carbon layer may obstruct light leaking from the waveguide and result in delamination of the carbon due to thermal stress. The presence of carbon nano-pillars increases the electrode surface area, improves interaction with cells and potentially leads to enhanced detection efficiency of dopamine. The second step was deposition of gold by ebeam evaporation to define the pseudo-reference electrode (RE) and contact pads. In order to prevent metallization of the pyrolytic carbon, a $4 \mu \mathrm{m}$ thick layer of negative photoresist (AZ nLOF 2020) was used as a mask layer completely covering the carbon during gold deposition. Using e-beam evaporation, $200 \mathrm{~nm}$ of gold with $20 \mathrm{~nm}$ titanium adhesion layer were deposited (Figure 2c). Metal lines were defined by lift-off of the photoresist in a bath containing Remover 1165 (maintained at $40{ }^{\circ} \mathrm{C}$ with ultrasonication for 3 hours). Contact pads were patterned with gold to ensure excellent electrical connection.

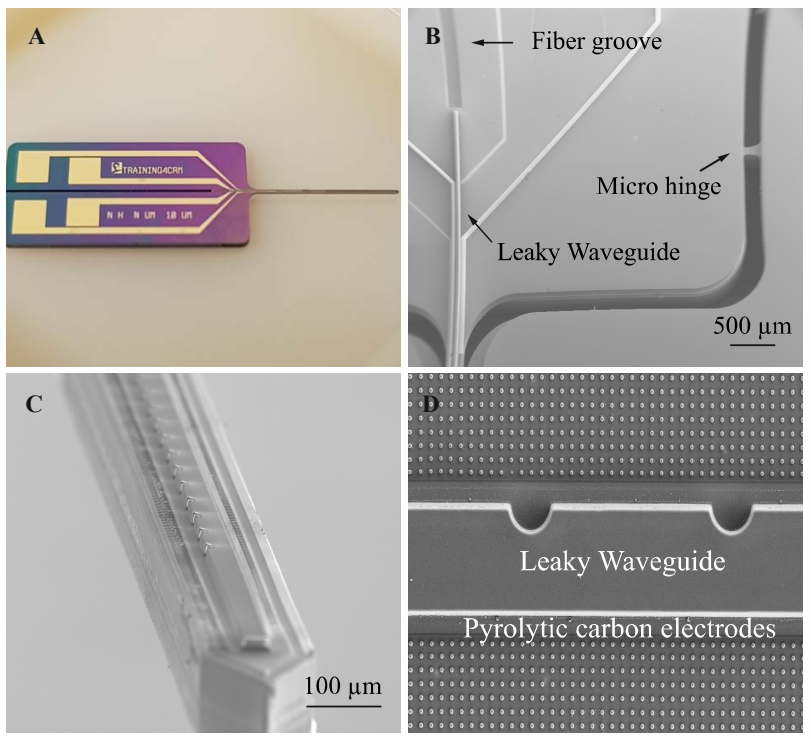

Figure 3: A: Final released optical neural probe containing pyrolytic carbon working and counter electrodes, gold pseudo-reference electrode and leaky $S U$ 8 waveguide. B: SEM image showing neural probe attached to the wafer with micro hinges. C: Released probe shank and tip showing the leaky part of the waveguide and electrodes. D: Close up of leaky waveguide surrounded by pyrolytic carbon electrodes with nano-pillars.

The wafer was subsequently etched $300 \mu \mathrm{m}$ from the backside with DRIE using fluorine chemistry (STS MESC Multiplex ICP). The backside etch depth determines the final thickness of the probe shank. Next, the leaky optical waveguide was patterned on the front side of the wafer. Following spin coating of a $20 \mu \mathrm{m}$ thick SU-8 layer (SU-8 $2035,120 \mathrm{~s}$ at $3600 \mathrm{rpm})$, UV lithography using a maskless aligner (dose of $250 \mathrm{mJcm}^{-2}$ ) was used to pattern the $40 \mu \mathrm{m}$ wide waveguide (Figure $2 \mathrm{~d}$ ). The baking processes were the same as described above. A large cross-section of the waveguide allows for better coupling of light, while at the same time increasing the width of the probe shank. Indentations in the waveguide structure were also patterned during lithography to obtain a leaky section. Finally, $50 \mu \mathrm{m}$ deep grooves were etched (DRIE) from the front side for releasing the probes (Figure $2 \mathrm{f}$ ). The wafer was mounted on a carrier wafer during the front side etching. The front side etch was also used to etch the groove for placement of an optical fiber to couple light into the waveguide. The probe shank was $7 \mathrm{~mm}$ long, $180 \mu \mathrm{m}$ wide and $50 \mu \mathrm{m}$ thick. Figure 3A shows released neural probes. Micro-hinges hold the neural probes in place and prevent them from 
detaching from the wafer during etching (Figure 3B). This allows the separation of neural probes from the wafer by the application of a small force breaking the hinge. A wider shank would accommodate more space for waveguide and electrodes but increases the tissue damage during initial implantation.

Figures $3 \mathrm{C}$ shows the released probe shank containing the leaky optical waveguide in the center surrounded by carbon with nano-pillars ( $28 \mu \mathrm{m}$ wide WE and $42 \mu \mathrm{m}$ wide $\mathrm{CE}$ ) and $14 \mu \mathrm{m}$ wide gold RE. Figure 3D shows the SEM of leaky optical waveguide surrounded by carbon nanopillars. The nano-pillars are around $300 \mathrm{~nm}$ tall and do not prevent the light from reaching cells.

\section{CHARACTERIZATION}

\section{Optical characterization}

Light propagation in a waveguide follows the principle of total internal reflection. The refractive indices of the core and the cladding define the critical angle $(\theta)$ for the propagation of light. The refractive index of SU-8 was measured to be 1.6. When implanted, cerebral spinal fluid (CSF) (refractive index 1.34 [9]) surrounds the waveguide. Since light has to leak uniformly over a large volume to stimulate the dopaminergic neurons in proximity of the waveguide, the waveguide design was simulated using the ray optics module of COMSOL. The $2 \mathrm{D}$ model simulates the propagation of rays in a waveguide. In a pristine waveguide, light propagates from one end to the other without excessively leaking from the sides. Introduction of some indentations in the waveguide structure changes the incidence angle locally. Rays with incidence angle smaller than the critical angle refract out of the core. Different designs of indentations in the waveguide were simulated to determine the optimal design for uniform light leakage. The final design consisted of a repeated semi-circular indentation in the waveguide structure (Figure 3D). The circular shape of the indentation allows light to leak out on both sides of the waveguide to illuminate neurons in the surrounding media. The type of cladding influences the distribution of light leakage. Air as the cladding $\left(\theta \approx 39^{\circ}\right)$ results in a wider spread of the leaking light transverse to the propagation direction (Figure 4A). The CSF cladding $\left(\theta \approx 59^{\circ}\right)$ guides the leaked light outwards in the propagation direction (Figure 4B). The leaked light carries most of the input power. This is desired to uniformly stimulate a large population of neurons surrounding the waveguide along its length.

The intensity of light leaking from the waveguide was measured using a power meter. Blue light at a wavelength of $460 \mathrm{~nm}$, which is the wavelength typically used for optogenetic stimulation of cells, from a laser diode (PL450B, Osram Opto Semiconductors Inc.) was coupled into a $50 \mu \mathrm{m}$ core diameter optical fiber (FG050UGA, Thorlabs Inc.). The other end of the cleaved optical fiber was placed in the groove on the topside of the neural probe coupling light into the SU-8 waveguide. The power of light leaking from the leaky waveguide was $7 \mu \mathrm{W}$ when the power at the end of the input optical fiber was $85 \mu \mathrm{W}(2 \mathrm{~ms}$ pulse, $10 \mathrm{~ms}$ period). This translates to an intensity of about $2 \mathrm{mWmm}^{-2}$ on one side of the waveguide, which is sufficient to activate ChR-2 having an activation threshold of $1 \mathrm{mWmm}^{-2}$ [10]. The spatial distribution of light is important to define the region of pyrolytic carbon for electrochemical detection of dopamine release. The waveguide was immersed in gelatin containing suspended green fluorescent nano-beads. Blue light leaking from the waveguide excites the fluorescent nano-beads thus revealing the distribution of light (Figure 4D). The presence of nano-pillars does not obstruct the path of the leaking light. Light leaks from both sides of the waveguide and the observed distribution correlates well with the simulation results.
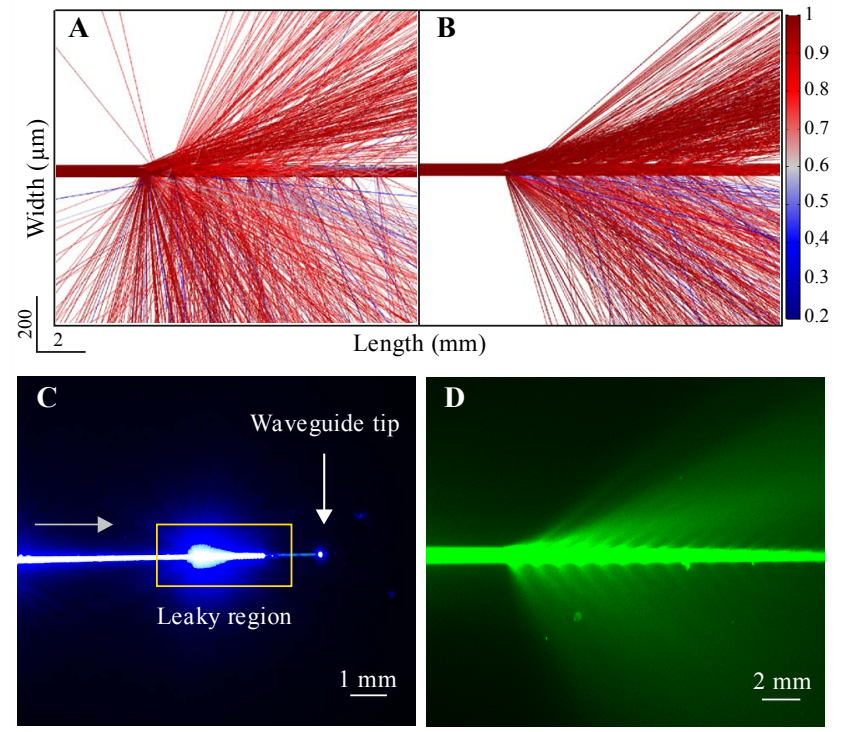

Figure 4. COMSOL ray tracing simulation showing distribution of light leaking from the waveguide. A: Air cladding. B: CSF cladding. C: Blue light at $460 \mathrm{~nm}$ coupled into leaky SU-8 waveguide (air cladding) showing light propagation and leakage. Most of the light is emitted in the leaky region resulting in very little light reaching the tip of the leaky waveguide. D: Fabricated leaky waveguide coated with fluorescent nano-beads revealing a pattern of light leakage similar to the simulation results.

\section{Electrochemical characterization}

Pyrolytic carbon derived from SU-8 has been widely used for electrochemical monitoring of cells $[9,10]$. We characterized the electrochemical behavior of the pyrolytic carbon electrodes by acquiring cyclic voltammograms $(\mathrm{CV})$ in $10 \mathrm{mM}$ hexacyanoferrate (II/III) $\left(\left[\mathrm{Fe}(\mathrm{CN})_{6}\right]^{2+/ 3+}\right)$ containing PBS. The electrodes patterned on the probe shank were used for acquiring the CVs shown in Figure 5. Oxygen plasma treatment renders the carbon layer hydrophilic and incorporates oxygen functional groups, which improves the wetting of the carbon surface. Moreover, the oxygen groups enhance the oxidation of dopamine at the electrode surface [8]. The CVs display semi-sigmoidal behavior, which is characteristic of microband electrodes, with peak separation $\left(\Delta E_{p}\right)$ of $300 \mathrm{mV}$ at a scan rate of $100 \mathrm{mVs}^{-1}$. This is in the same range as previously reported for SU-8 derived pyrolytic carbon [13]. Oxidation and reduction peak currents start to appear at higher scan rates. We also observed widening of the $\Delta \mathrm{E}_{\mathrm{p}}$ $\left(300 \mathrm{mV}\right.$ at $100 \mathrm{mVs}^{-1}$ to $450 \mathrm{mV}$ at $900 \mathrm{mVs}^{-1}$ ) with increasing scan rates due to the quasi-reversible reaction kinetics. 

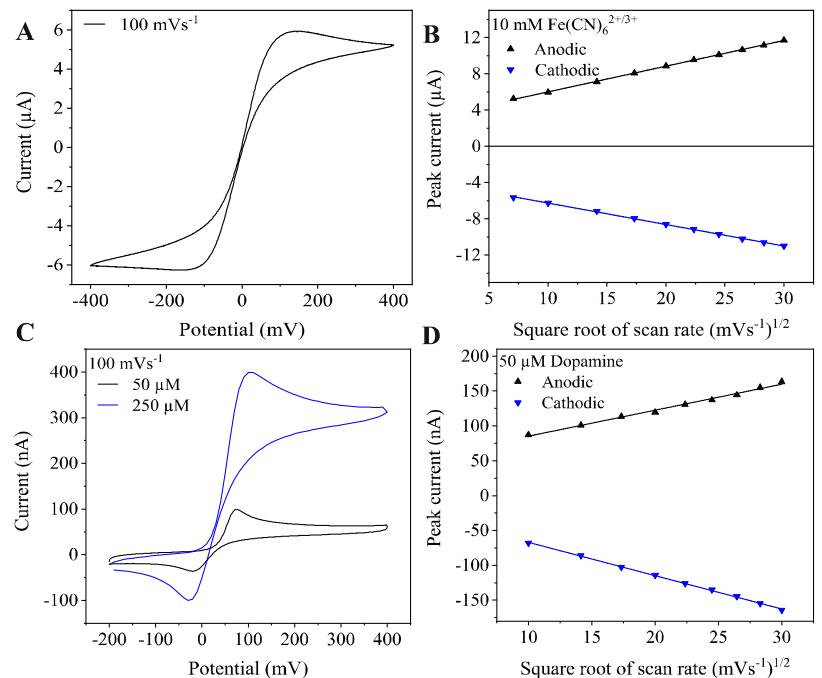

Figure 5: A) Cyclic voltammogram of neural probes acquired in $10 \mathrm{mM}\left[\mathrm{Fe}(\mathrm{CN})_{6}\right]^{2+/ 3+}$ at $100 \mathrm{mVs}^{-1}$. B) Plot of anodic and cathodic peak current versus square root of scan rate showing linearity up to $900 \mathrm{mVs}^{-1}$. C) Comparison of CVs acquired at $100 \mathrm{mVs}^{-1}$ in dopamine solution at two different concentrations (50 $\mu \mathrm{M}$ and 250 $\mu M)$. D) Anodic and cathodic peak currents follow a linear relationship with the square root of scan rate.

Since we intend to detect dopamine released by neurons, we acquired $\mathrm{CVs}$ at different concentrations of dopamine in PBS and at different scan rates. As shown in Figure $5 \mathrm{C}$, the CVs display higher anodic peak currents compared to the cathodic peak currents. This behavior has previously been observed for measurement of dopamine with pyrolytic carbon electrodes [14] and is due to the quasi-reversible nature of dopamine electrochemistry. The peak separation for $50 \mu \mathrm{M}$ dopamine at $100 \mathrm{mVs}^{-1}$ was 90 $\mathrm{mV}$. We noticed a slight widening of the peaks with increasing scan rates. In both cases, the anodic and cathodic peak currents follow a linear relationship for square root of scan rates as high as $900 \mathrm{mVs}^{-1}$, which is characteristic for diffusion-controlled electrochemical reactions and indicates suitability of the pyrolytic carbon electrodes for dopamine detection.

\section{CONCLUSION}

In this work, opto-electrical neural probes consisting of a SU-8 leaky optical waveguide and pyrolytic carbon electrodes integrated on a $180 \mu \mathrm{m}$ wide and $50 \mu \mathrm{m}$ thick probe shank were developed and characterized for potential in vivo optogenetic application. The optical waveguide was designed to be leaky for more than $2 \mathrm{~mm}$ in length by the introduction of indentations in the waveguide structure. These indentations locally modified the condition for total internal reflection and caused the light to refract out from both sides of the waveguide. Uniform leakage of light ensures illumination of optogenetically modified stem cells surrounding the waveguide. The waveguide design was initially simulated and subsequently fabricated by UV photolithography. Using fluorescent nanoparticles, we were able to visualize the distribution of blue light leaking from the waveguide. The observed light distribution was in good agreement with the simulation results. Moreover, the intensity of light leaking from one side of the waveguide was measured to be $2 \mathrm{mWmm}^{-2}$ for $2 \mathrm{~ms}$ laser pulses, which is sufficient to stimulate the ChR-2 expressing cells. Finally, the electrochemical properties of the pyrolytic carbon are suitable for dopamine measurements and in agreement with the literature. This study shows the potential of this device for in-situ optical stimulation of neurons and electrochemical detection of neurotransmitter release.

In the future, we will culture and differentiate optogenetically modified human neural stem cells on the neural probes for real-time electrochemical detection of dopamine released upon optical stimulation.

\section{ACKNOWLEDGEMENTS}

This project was funded by the European Union Horizon 2020 Programme (H2020-MSCA-ITN-2015 and H2020-MSCA-ITN-2016) under the Marie SkłodowskaCurie Innovative Training Network, projects BrainMatTrain and Training4CRM Grant Agreement Nos. 676408 and 722779 , respectively.

\section{REFERENCES}

[1] E. Arenas et al., Dev. 142(11), pp 1918, 2015.

[2] C. W. Olanow et al., Lancet Neurol 5(8), pp 677, 2006.

[3] G. Nagel et al., Proc. Natl. Acad. Sci. U. S. A. 100(2), pp 13940, 2003.

[4] J. A. Steinbeck et al., Nat. Biotechnol. 33(2), pp 204, 2015.

[5] A. N. Zorzos et al., Opt. Lett. 35(24), pp 4133, 2010.

[6] T. V. F. Abaya et al., Biomed. Opt. Express 3(12), pp 3087, 2012.

[7] F. Pisanello et al., Neuron 82(6), pp 1245, 2014.

[8] L. Amato et al., Adv. Funct. Mater. 24(44), pp 7042, 2014.

[9] J. Sun et al., Opt. Express 20(2), pp 1084, 2012.

[10] F. Zhang et al., Nat. Methods 3(10), pp 785, 2006.

[11] L. Amato et al., Carbon N. Y. 94, pp 792, 2015.

[12] Y. M. Hassan et al., ECS Trans 72(1), pp 35, 2016.

[13] S. Hemanth et al., ECS Trans 72(2), pp. 117, 2016.

[14] S. Vasudevan et al., Adv. Sci., pp. 1902011, 2019.

\section{CONTACT}

*S.S.Keller, Tel: +45 45255846; suke@dtu.dk 